\title{
Síntesis y copolimerización de poliimidas a partir de aminas aromaticas mediante irradiación por microondas
}

\author{
Synthesis and Copolymerization of Polyimides From Aromatic Amines by \\ Microwave Irradiation
}

\author{
Diana Lorena Pérez Bernal ${ }^{\mathrm{a}}$ \\ Efrén de Jesús Muñoz Prieto ${ }^{{ }^{*}}$ \\ Joaquin Palacios-Alquisirac \\ Carlos Antonio Rius-Alonso ${ }^{\mathrm{c}}$
}

Recepción: 09-nov-2015

Aceptación: 07-may-2016

\section{Resumen}

Se sintetizo la amina aromática (4,4'-(4,4'-isopropilidendifenil-1-1'-diioxi) dianilina), con el anhídrido 4,4' (4,4'-(isopropilidendifenoxi) bis (anhídrido ftalico) (IDBPA)), para obtener una poliimida a la cual por medio de irradiación de microondas se le injerto a la cadena principal el monómero $\varepsilon$-caprolactama. La poli (caprolactama) obtenida tiene buenas características terminas de acuerdo a los resultados de DSC Y TGA. La poliimida se caracterizó por las técnicas analíticas, espectroscopia infrarroja de transformada de Fourier (FTIR), análisis termogravimétrico (TGA), calorimetría diferencial de barrido (DSC) y resonancia magnética nuclear ${ }^{1} \mathrm{HRMN}$.

Palabras clave: lactamas, poliimidas, radiacion de microondas.

\begin{abstract}
The aromatic amine (4, 40- (4, 40-isopropylidene-diphenyl-1-10-diyldioxy) dianiline), was synthesized with the anhydride 4, 40(4, 40 (isopropylidene-diphenoxy) bis (phthalic anhydride) (IDBPA)), to obtain a polyimide which by microwave irradiation was grafted to the backbone a Caprolactam monomer. The poly (Caprolactam) obtained has good finish characteristics according to DSC and TGA results. The polyimide was characterized by instrumental techniques such as; Fourier Transform Infrared Spectroscopy (FTIR), Thermo Gravimetric Analysis (TGA), Differential Scanning Calorimetry (DSC) and Nuclear Magnetic Resonance, 1HNMR.
\end{abstract}

Key words: Lactamas, Poliimidas, Radiacion de microondas.

\footnotetext{
${ }^{a}$ Universidad Pedagógica y Tecnológica de Colombia, Tunja, Boyacá, Colombia.

${ }^{b}$ Faculta de Ciencias Químicas, Universidad Pedagógica y Tecnológica de Colombia, Tunja, Boyacá, Colombia.

*Autor de correspondencia: efren17@gmail.com

${ }^{\mathrm{c}}$ Facultad de Química de la Universidad Nacional Autónoma de México, UNAM, México, D. F.
} 


\section{Introducción}

En el trascurso del tiempo se ha investigado a escala de laboratorio e industrial varias metodologías para el mejoramiento, transformación y producción de nuevos materiales útiles para el desarrollo de la humanidad obteniendo materiales poliméricos renovables indispensables en la creación de una sociedad sostenible. Uno de los temas con mayor impacto es reducir el consumo de energía y solventes en procesos de síntesis de polímeros. Con la aplicación de la irradiación de microondas se pueden reducir estas variables ya que ofrecen varias ventajas comparadas con las metodologías convencionales de las cuales se pueden nombrar: reducción del tiempo de reacción y del consumo energético, rendimiento y pureza del producto obtenido y la posibilidad de no utilizar solventes.

La aplicación de las técnicas en la obtención de poliimidas, uno de los materiales más importantes en la industria de polímeros de ingeniería, de más alto rendimiento y de gran demanda, con una excelente estabilidad térmica, buena resistencia química, así como altas propiedades eléctricas y propiedades mecánicas elevadas que cumplen con los requisitos de diversas aplicaciones industriales, tales como su uso en aislamiento electrónico, piezas de automóviles, transporte y materiales de aviación, materiales útiles en microelectrónica, materiales compuestos y así sucesivamente [1]. Sin embargo, estos poliimidas a menudo tienen una baja solubilidad en los disolventes comunes y tienen altas temperaturas de reblandecimiento, debido a la estructura rígida de su cadena principal y fuertes interacciones entre las cadenas, características que resultan de los grupos altamente simétricos y altamente polares, por lo que su procesamiento es difícil o demasiado caro para ser comercialmente viable [2]. La técnica más común utilizada para fabricar poliimidas solubles es utilizar un poli (ácido ámico) que después se convierte a una poliimida junto con el método de preparación de la PI por la reacción de anhídridos con aminas [3].

Desde hace varias décadas, se han hecho muchos esfuerzos para superar grandes inconvenientes y modificar las interacciones moleculares para permitir el procesamiento por técnicas convencionales, tales como el procesamiento de masa fundida, introducción de enlaces flexibles, átomos de flúor, grandes grupos colgantes y unidades asimétricas en colum- nas vertebrales de poliimida, mientras se mantiene la estabilidad termo-oxidativa del polímero [4]. Estos estudios han implicado tres grandes modificaciones estructurales: la incorporación de los vínculos térmicamente estables pero flexibles o asimétricos en la cadena principal, la introducción de grandes sustituyentes colgantes polares o no polares a la cadena de polímero, la interrupción de la simetría y la recurrencia de regularidad mediante la copolimerización. Por ejemplo, la incorporación de enlaces flexibles, tales como -O-, - $\mathrm{CH}_{2^{-}},-\mathrm{SO}_{2}-\mathrm{y}$ grupos hexafluorisopropiliden en la cadena principal que disminuyen la rigidez de la cadena principal del polímero e inhiben el empaquetamiento de las cadenas, lo que reduce las interacciones entre cadenas y conduce a mejorar la solubilidad. La incorporación geométrica o molecular de un dianhídrido asimétrico o diamina y componentes en la cadena principal de poliimida es uno de los enfoques más exitosos en la consecución de la solubilidad sin sacrificar sus excelentes propiedades, estas modificaciones estructurales de monómeros también han dado lugar a nuevas poliimidas con varias propiedades mejoradas. La necesidad de solubilizar estos compuestos es para facilitar su explotación en la fabricación de dispositivos ópticos, películas, fibras, materiales dieléctricos y semiconductores, para fines biomédicos y en inhibición de la corrosión [5]. Ultem $\AA$ (polieterimida, PEI) (General Electric Co.) es un buen ejemplo de un polímero que se procesa fácilmente, tiene propiedades mecánicas deseables y retiene propiedades térmicas.

Las PI han sido sintetizadas por varios autores como en la reacción de una poli (éter imida) (PEIs) de color claro y buenas propiedades mecánicas, obtenida a partir de 2,5-bis (3,4-dicarboxifenil) bifenilo y diversos éteres-diaminas aromáticas a través de una técnica de polimerización convencional de dos etapas que incluye poliadición de apertura de anillo a temperatura ambiente para formar el poli (ácido ámico) (PAA), seguido de una imidación térmica [6] Descripción de la preparación de una serie de novedosos perfluoroalquilenos y poliimidas aromáticas unidas por éter, dependiendo de la naturaleza del precursor diamina, se prepararon las poliimidas por punto de fusión o técnicas de solución, este último a través de la poli (ácidos ámicos correspondiente), los polímeros se caracterizaron por una variedad de métodos y su estructura se discute [7]. La reacción de transimidización se ha utilizado con éxito para 
preparar una serie de copolímeros de bloque segmentados usando monómeros que contienen los anillos de imida en el que una reacción incorpora una diimida con una diamina a través de un catalizador básico para inducir una reacción de intercambio debido a la presencia de dos extremos reactivos [8]. La polimerización de un dianhídrido ciclobutanotetracarboxílico o biciclo [2.2.1] heptano-2-metano carboxilico-3,5,6-tricarboxílico-2,3: 5,6-dianhídrido con diversas diaminas alicíclicas $\mathrm{N}$-sililado se llevaron a cabo con $\mathrm{N}, \mathrm{N}$-dimetilacetamida (DMAc) a temperatura ambiente, dando un poli (ácido ámico trialquilsiliester) como polímero precursor que se hidrolizó a poli (ácido ámico), obteniendo poliimidas totalmente alicíclicas con altos pesos moleculares [9] y la reacción de un monómero del AB que contiene una amina aromática en un extremo y el éster orto del ácido carboxílico en el otro extremo usando un catalizador fosforado para formar el éster de la poliamida que se convierte posteriormente a PI [10].

La tecnología de microondas se ha utilizado en la síntesis orgánica desde 1986. Esta tecnología se ha desarrollado rápidamente en el campo del análisis, la síntesis química y es convertido en un componente importante en la química combinatoria y verde. La aplicación de irradiación de microondas para proporcionar la energía de activación en algunas reacciones orgánicas se pueden acelerar dramáticamente y completarse en unos pocos minutos y los rendimientos se han mejorado en comparación con los de los métodos convencionales ya que su calentamiento es más homogéneo [11]. Además, puede promover la nucleación y reducir significativamente el tiempo de síntesis y ofrecen numerosas ventajas como el calentamiento sin contacto (reducción del sobrecalentamiento de las superficies de materiales), transferencia de energía en lugar de transferencia de calor (radiación penetrante), material selectivo y volumétrico, la etapa de iniciación rápida igual que la de su parada y un gradiente inverso es en forma de calor comienza a acumularse desde el interior (núcleo) del cuerpo material hasta el exterior. En consecuencia, no sólo el problema de la contaminación se puede superar, pero el riesgo de sobrepresión y explosiones también se puede evitar. Ha habido algunos informes sobre el uso de la irradiación de microondas para la síntesis de PI. Ejemplos recientes incluyen poli (éster imida) y poli (amida imida) a partir de cloruros de diácido y PI por dianhídridos y diaminas [3].
El siguiente trabajo describe una metodología limpia, en el cual se obtiene poliimidas a partir de dos aminas y un dianhídrido mediante la aplicación de la irradiación de microondas como fuente de activación de la reacción, obteniendo altos rendimientos, tiempos de reacción cortos y la obtención de materiales biodegradables y en algunos casos estos pueden reemplazar a otros más contaminantes difíciles de degradar como pueden ser los metales. Simultáneamente se caracterizan los polímeros obtenidos con las técnicas instrumentales de espectrofotometría de infrarrojo (FTIR), ${ }^{1} \mathrm{HRMN}$, análisis térmico DSC y TGA.

\section{Metodología}

Materiales: $\quad 4^{\prime}-\left(4,4^{\prime}\right.$-(isopropilidendifenoxi) bis (anhidrido ftalico) (IDBPA), 4,4'-(4,4'-isopropilidendifenil-1, $1^{\prime}$-diioxi) dianilina, $\varepsilon$-caprolactama, Litio bis(trimetilsilil) amida $1.0 \mathrm{M}$ en THF, Sigma Aldrich, las propiedades de estos reactivos se describen en la tabla 1.

Síntesis de poliimidas: reacciones en masa: se adicionaron $(5 \mathrm{mmol})$ de anhídrido en un vaso de precipitado de $50 \mathrm{ml}$ se fundió a $200{ }^{\circ} \mathrm{C}$ y se agregaron ( $5 \mathrm{mmol}$ ) de amina aromática, se agito fuertemente ya que la reacción es rápida, formando un polímero oscuro de consistencia dura. La reacción tuvo un tiempo de $15 \mathrm{~min}$. Después de este tiempo las muestras se dejaron en la estufa al vacío durante toda la noche a una temperatura de $80^{\circ} \mathrm{C}$. Metodología adaptada por los autores del presente trabajo a partir de la Ref. 12. Correspondiente a los autores G. s. Hans R.Kricheldorf, Shu-ching Fan [12].

Preparación de copolímeros de injerto: el ambiente en que se trabaja es bajo una atmosfera inerte. Se hace la disolución con una relación 8:2 $(0,8 \mathrm{~g}$ de $\varepsilon$-caprolactama y $0,2 \mathrm{~g}$ de PIs-A 4$)$ y $9: 1(0,9 \mathrm{~g}$ de $\varepsilon$-caprolactama y $0,1 \mathrm{~g}$ de PIs-A 4 ) a $180{ }^{\circ} \mathrm{C}$, hasta que se disuelve totalmente el polímero, el tiempo de cada reacción fue de $1 \mathrm{~h}$, se le agrega $0.9 \mathrm{~mL}$ del activador litio bis (trimethylsylic) amida $1.0 \mathrm{M}$ para que las concentraciones queden equivalentes. Los sólidos obtenidos se enfrían lentamente a temperatura ambiente a una velocidad de $2.5^{\circ} \mathrm{C} / \mathrm{min}$. Todos los copolímeros obtenidos se convierten en trozos pequeños y luego polvo fino para sus futuros análisis. 
Tabla 1. Características fisicoquímicas de los reactivos.

\begin{tabular}{lcccccc}
\hline Nombre & Formula & $\mathrm{mp}^{\circ} \mathrm{C}$ & $\mathrm{bp}^{\circ} \mathrm{C}$ & $\mathrm{PM} \mathrm{g} / \mathrm{mol}$ & Pureza & Estado \\
\hline $\begin{array}{l}4,4^{\prime}-\left(4,4^{\prime}-\right. \\
\text { (isopropilidendifenoxi) }\end{array}$ & $\mathrm{C}_{31} \mathrm{H}_{20} \mathrm{O}_{8}$ & $184-187$ & - & 520,5 & 97 & Sol \\
bis(anhidrido \\
ftalico) (IDB-
\end{tabular}

Caracterización: Los espectros infrarrojos se realizaron con un espectrofotómetro (FTIR) modelo 1605 marca Perkin-Elmer. El análisis de Termogravimétrico (TGA) y la calorimetría de exploración diferencial (DSC) se determinaron con un termoanalizador con módulos de calorimetría y gravimetría modelos DSC821 ${ }^{e}$ y un TGA/SDTA851 ${ }^{e}$ MettlerToledo, con una velocidad de calentamiento de 10 ${ }^{\circ} \mathrm{C} /$ minuto en atmósfera de $\mathrm{N}_{2}$ en un rango de temperatura de $0-450{ }^{\circ} \mathrm{C}$. Ambos son de la marca MettlerToledo. La viscosidad intrínseca de polímeros se analizó usando un viscosímetro de Ubbelohde, marca Canon, en un baño de agua. La solubilidad de las muestras del poliimidas se estudió disolviendo el polímero aproximadamente $0,050 \mathrm{mg}$ en tres gotas de cada solvente a una temperatura de $23{ }^{\circ} \mathrm{C}$.

Reacción de polimerización: Las reacciones de la polimerización fueron realizadas en un horno mono modal modelo Discovery Explorer Hybrid marca CEM con control de temperatura y potencia en un intervalos de 0-300 watts. En un tubo de vidrio del reactor de $10 \mathrm{~mL}$ con una mezcla equimolar del dianhídrido y diamina.

\section{Resultados y discusión}

Para identificar la composición de los reactivos de partida se realizaron los espectros infrarrojos que se muestran en la tabla 2, el espectro del anhídrido muestra las bandas 1847 y $1770 \mathrm{~cm}^{-1}$ correspondientes a los enlaces $\mathrm{C}=\mathrm{O}$ característicos de estos grupos funcionales y las bandas de la amina aromática como un doblete en el rango de $3400-3500 \mathrm{~cm}^{-1}$, la absorción de la tensión N-H aparece entre 3423 y
$3333 \mathrm{~cm}^{-1}$ y de flexión sobre $1494 \mathrm{~cm}^{-1}$, de acuerdo a esto los reactivos están en las condiciones aptas para la síntesis.

Síntesis de poliimidas: la ruta más versátil para la obtención de poliimidas aromáticas implica la reacción de un dianhídrido tetracarboxílico aromático con una diamina aromática para formar inicialmente un precursor, con una configuración no ordenada, ácido de poliimida (ácido poliámico). El polímero precursor puede ser química o térmicamente convertido a poliimida. Como un ejemplo, 4,4'-(4,4' -(isopropilidendifenoxi)bis(anhidrido ftalico) se representa para reaccionar con $4,4^{\prime}-\left(4,4^{\prime}\right.$ isopropilidendifenil-1 $11^{\prime}$-diioxi) dianilina y obtener el ácido poliámico de alto peso molecular que se forma fácilmente por la apertura del anillo debido al ataque nucleofílico de un grupo amino en el grupo carbonilo del anhídrido y así dar paso a la formación de la poliimida.

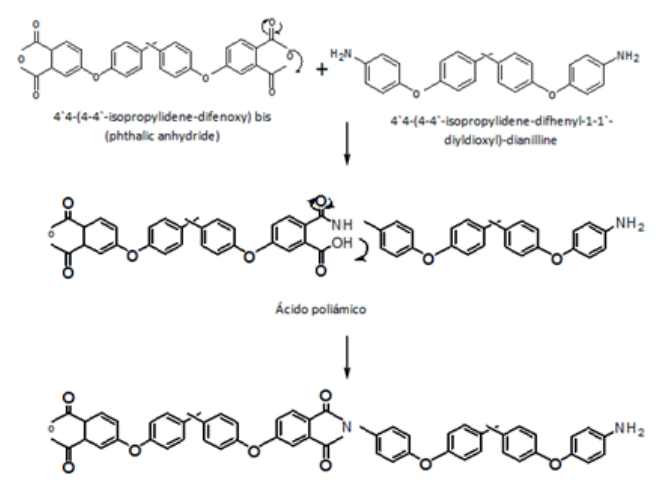

Figura 1. Mecanismo de reacción de obtención de poliimida. 
Tabla 2. Espectros infrarrojos de materias primas.

(15)<smiles>[CH]N([Si](C)(C)C)[Si](C)(C)C</smiles>

Litio bis(trimetilsilil) amida $1.0 \mathrm{M}$ en THF

En base en la metodología antes descrita se logró obtener una poliimida de color café oscuro, el cual para someterla a caracterización por espectroscopia infrarroja se pulverizo para su análisis, el espectro infrarrojo mostro las siguientes bandas características figura 3. El espectro exhibe bandas característica de absorción del ácido poliámico en torno a 2900-3400 $\mathrm{cm}^{-1}$ para el grupo N-H y el grupo O-H $(\mathrm{COOH})$ respectivamente, bandas en $1718 \mathrm{~cm}^{-1}$ son debido a los tramos asimétricos y simétricos de grupo carbonilo de imida [13], se desaparece la banda 1847 $\mathrm{cm}^{-1}$ característica del anhídrido lo que quiere decir que reacciono para formar el ácido ámico, de la misma manera las bandas de la amina en el rango de $3400-3200 \mathrm{~cm}^{-1}$ también desaparecieron, la poliimida sintetizada presenta sus principales bandas exactamente en la regiones esperadas como se describe en la literatura [14] y [15] teniendo en cuenta estas alteraciones indican que si se obtuvo la poliimida esperada.

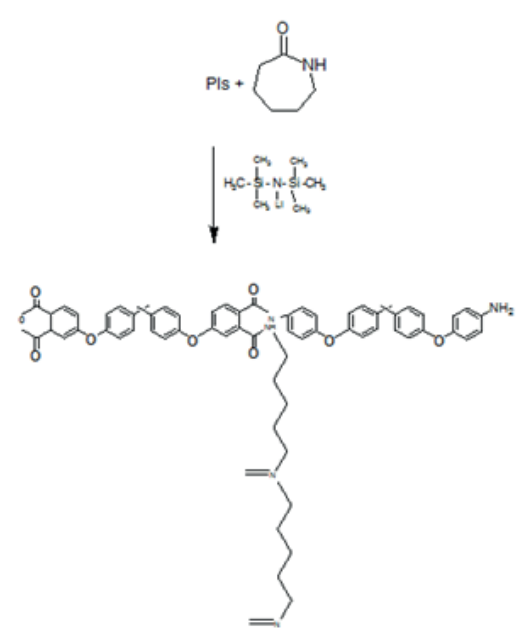

Figura 2. Reacción final de copolimerización de injerto.

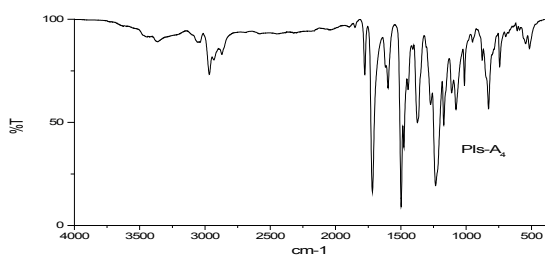

Figura 3. Muestra PIs- $\mathrm{A}_{4}, 5 \mathrm{mmol}$ de anhídrido y 5 mmool de amina aromática, tiempo de reacción 15 minutos, sin solvente. 
Copolimerización de poliimidas: en la figura 2 se ilustra la reacción de injerto del monómero $\varepsilon$-caprolactama sobre la cadena polimérica de la poliimida obtenida, una de las funciones del activador Litio bis (trimetilsilil) amida 1.0 M en THF es de abrir el anillo de la poliimida para formar el copolímero.

Para confirmar la estructura se hace el análisis de ${ }^{1}$ HRMN de la poliimida obtenida, una señal característica de la parte aromática se observó en el intervalo de 6.96 a 7.88 ppm (figura 4), se apareció el pico de absorción de protones de los metilos en 1.75 y $1.69 \mathrm{ppm}$, también se observaron las señales en 3.76 ppm, 3.72 ppm y 1.87 ppm, 1.85 ppm indican que el activador se pegó a la estructura del polímero confirmando su función en la ayuda de la apertura de anillo de la poliimida.



Figura 4. Espectro ${ }^{1} \mathrm{HRMN}$ de PIs- $\mathrm{A}_{4}$.

Para la copolimerización las poliimidas se disolvieron en $\varepsilon$-caprolactama fundida a diferentes concentraciones y a una temperatura de $180^{\circ} \mathrm{C}$ en el microondas, esto para que los aniones ataquen los enlaces imida para generar el inicio de la polimerización de la $\varepsilon$-caprolactama, el activador actúa en la apertura del anillo de $\varepsilon$-caprolactama y por tanto genera copolímeros de injerto [16], en la figura 5-a) injerto con un tiempo de reacción 5 minutos, $\mathbf{g A}_{8}-\mathbf{5}$ (8:2) y $\mathbf{g A}_{9}-\mathbf{5}$ (9:1), el espectro mostros absorciones fuertes relacionadas con las bandas $\mathrm{C}-\mathrm{H}$ aparecen en 2929 y $2858 \mathrm{~cm}^{-1}$ y uno más débil en $1462 \mathrm{~cm}^{-1}$, las absorciones $\mathrm{N}-\mathrm{H}$ y $\mathrm{C}=\mathrm{O}$ son las más fuertes, características de la función de amida y aparecen en 3297 y $1636 \mathrm{~cm}^{-1}$, se puede ver otro pico de absorción fuerte en $1540 \mathrm{~cm}^{-1}$ y uno bien definido pero la absorción débil se producen $3074 \mathrm{~cm}^{-1}$ y figura 5-b) injerto con un tiempo de reacción 30 minutos, $\mathbf{g A N}_{8}$ 30 (8:2) y $\mathbf{g A N}_{9}-\mathbf{3 0}$ (9:1), en ambos espectros se observan las bandas características de las poliimidas injertadas, la ausencia de las bandas en el rango de $1800 \mathrm{~cm}^{-1}$ confirma la ausencia de reacción del anhídrido queriendo decir que la conversión de la imida se llevó a cabo ya que en este rango aparecen bandas debido al estiramiento asimétrico del anhídrido.
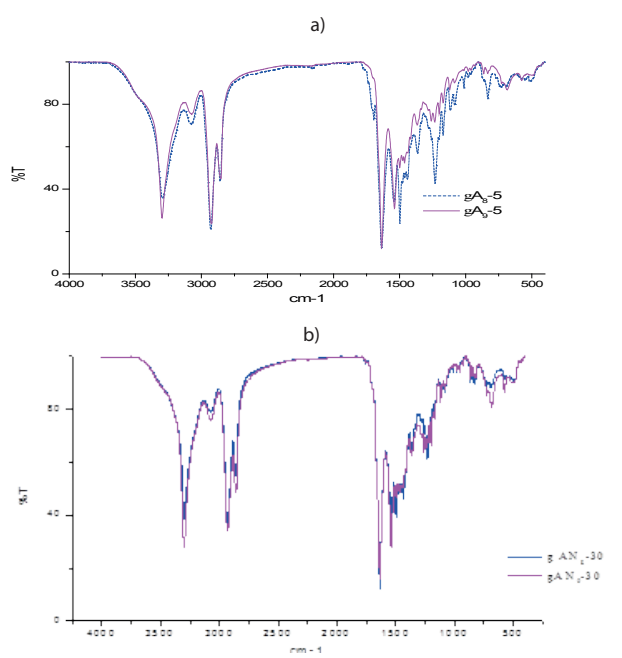

Figura 5. Injerto del monómero $\varepsilon$-caprolactama en la cadena polimerica en diferentes concentraciones a) $\mathbf{g A}_{8}$ 5 (8:2) y $\mathbf{g A}_{9}-\mathbf{5}$ (9:1); b) $\mathbf{g A N}_{8}-\mathbf{3 0}$ (8:2) y $\mathbf{g A N}_{9}-\mathbf{3 0}$ (9:1).

\section{Análisis térmico de la reacción de injerto por} activación con irradiación de microondas: En las curvas de TGA de $\mathrm{gA}_{8}-5$ y $\mathrm{gA}_{9}-5$, se puede observar que la pérdida de peso inicia a una temperatura de $95{ }^{\circ} \mathrm{C}$ y finaliza a $220{ }^{\circ} \mathrm{C}$, la curva con pendiente pequeña generalmente corresponde a la degradación de la cadena principal, las dos graficas que pertenecen a dos concentraciones diferentes del monómero $\varepsilon$-caprolactama son muy similares, figura 6 .

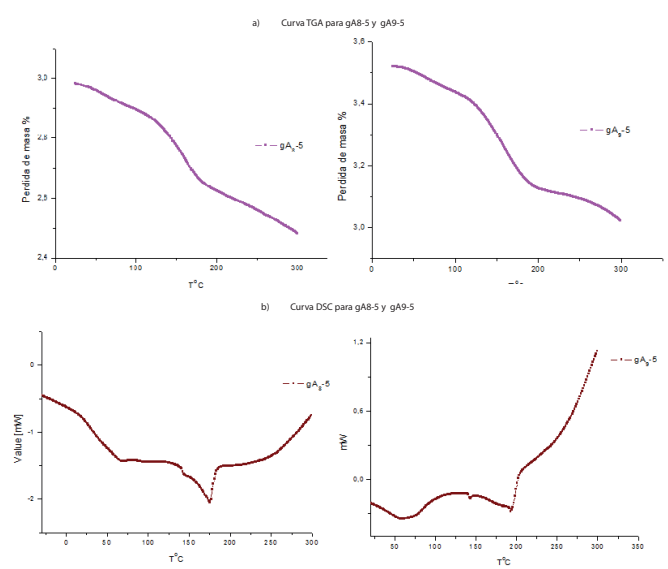

Figura 6. Análisis térmico a) curva TGA de $\mathrm{gA}_{8}-5 \mathrm{y}$ $\mathrm{gA}_{9}-5$, respectivamente, b) curva DSC de $\mathrm{gA}_{8}-5$ y gA 9 5 , respectivamente. 


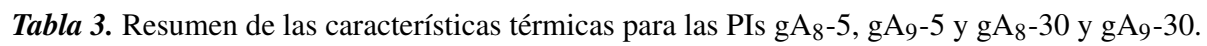

\begin{tabular}{|c|c|c|c|c|c|c|}
\hline \multirow[t]{2}{*}{ Poliimida } & \multirow[t]{2}{*}{$\begin{array}{l}\text { Proporciones mola- } \\
\text { res }(\varepsilon \text {-caprolactama: } \\
\text { PIs-A4 })\end{array}$} & \multicolumn{2}{|l|}{ TGA } & \multicolumn{3}{|c|}{$\begin{array}{l}\text { DSC (temperatura de } \\
\text { inicio de los procesos } \\
\text { endotérmicos }{ }^{\circ} \mathrm{C} \text { ) }\end{array}$} \\
\hline & & $\begin{array}{l}\text { (temperatura de inicio } \\
\text { de pérdida de peso }{ }^{\circ} \mathrm{C} \text { ) }\end{array}$ & $\operatorname{Td}\left({ }^{\circ} \mathrm{C}\right)$ & $1^{\circ}$ & $2^{\circ}$ & $3^{\circ}$ \\
\hline $\mathrm{gA}_{8}-5$ & $8: 2$ & 95 & 260 & 75 & 140 & - \\
\hline $\mathrm{gA}_{9}-5$ & $9: 1$ & 95 & 260 & 81 & 142 & 192 \\
\hline $\mathrm{gA}_{8}-30$ & $8: 2$ & 110 & 250 & 50 & 186 & - \\
\hline $\mathrm{gA}_{9}-30$ & $9: 1$ & 95 & 260 & 70 & 187 & - \\
\hline
\end{tabular}

En las curvas de TGA de $\mathrm{gA}_{8}-30$ se puede observar que la pérdida de peso inicia a una temperatura de $110{ }^{\circ} \mathrm{C}$ y finaliza a $220{ }^{\circ} \mathrm{C}$ y en gA9-30 la perdida de peso inicia a $95^{\circ} \mathrm{C}$ finalizando a $230^{\circ} \mathrm{C}$, la degradación de la cadena principal del polímero se diferenció por la cantidad de tiempo de reacción en el injerto, figura 7. En las curvas de DSC para el $g A_{8}-5$ se observaron dos picos endotérmicos: el primero a $75^{\circ} \mathrm{C}$ está cerca a la temperatura de inicio de pérdida de peso, el segundo bien definido empezó a $140{ }^{\circ} \mathrm{C}$ que se ve asociado con el inicio de la degradación del esqueleto principal del polímero (o pico exotérmico) y la temperatura máxima que continua hasta la descomposición, en la curva de $\mathrm{gA}_{9}-5$ también se evidencia a $81^{\circ} \mathrm{C}$ una endoterma que está relacionada con el inicio de pérdida de peso, a 142 ${ }^{\circ} \mathrm{C}$ una endoterma no muy definida y por ultimo a $192{ }^{\circ} \mathrm{C}$ inicio la degradación de la cadena principal.

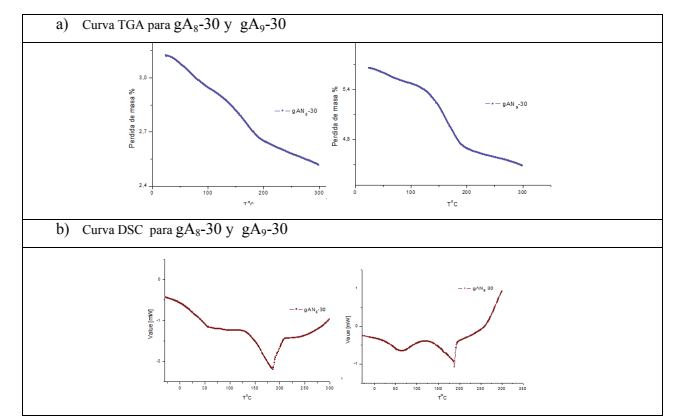

Figura 7. Análisis térmico a) curva TGA de $\mathrm{gA}_{8}-30 \mathrm{y}$ $\mathrm{gA}_{9}-30$, respectivamente, b) curva DSC de $\mathrm{gA}_{8}-30$ y $\mathrm{gA}_{9}-30$, respectivamente.

La tabla 3 resume las propiedades térmicas del injerto de poliimida en el cual se evalúa la estabilidad térmica de la PI según los valores de $\mathrm{Tg}$ la $\mathrm{gA}_{8}-5$ y gA $_{9}-5$ tiene baja movilidad de las cadenas, su nivel de deformación es restringido, presentan una temperatura de descomposición entre 250-260, por todo lo anterior la PI tiene una buena estabilidad térmica.
La calorimetría diferencial de barrido de todas las PIs muestra la degradación de diferentes concentraciones, éstos tienen más números de picos de degradación o tienen diferentes valores de energía para la degradación, a partir de la figura 5 b) y figura 6b) podemos resumir la información como la temperatura de inicio de la descomposición (o pico exotérmico) y la temperatura máxima que continúa hasta la descomposición, la duración total de la temperatura y de la degradación de la energía liberada durante estos descomposición, [17-19] características que se muestran en la tabla 3.

\section{Conclusiones}

Poliimida PIs $\mathrm{A}_{4}$ derivada de la amina aromática y el anhídrido se sintetizaron con éxito. Las estructuras fueron confirmadas por las técnicas analíticas descritas, las propiedades térmicas de la poliimida se determinaron mediante DSC y TGA, en el cual la temperatura final de descomposición es independiente de tiempo de reacción del injerto, de acuerdo a la concentración de momero la relación de 9:1 hace que el tiempo final de degradación sea mayor, debido a la poca cantidad de poliimida injertada en la cadena principal. La poliimida mostró buenas propiedades térmicas se deben a su estructura rígida de la fracción de diamina y dianhídrido. Es posible utilizar poliimidas durante períodos prolongados de tiempo a temperaturas de hasta $200{ }^{\circ} \mathrm{C}$, Se obtuvo un material de color café oscuro de consistencia muy dura y resistente. Estas características permiten a las poliimidas tener diversas aplicaciones en muchos campos.

\section{Referencias}

[1] E. Muñoz Prieto, J. Palacios Alquisira, C. Rius Alonso, "Microwave and Ultrasound Activation Effect on Cationization of Corn and Potato 
Starches", Ciencia en Desarrollo, vol. 4, no.1, pp. 151-173, 2012.

[2] DJ Liaw, KL Wang, YC Huang, KR Lee, JY Lai, CS Ha, . ${ }^{A}$ dvanced polyimide materials: syntheses, physical properties and applications", Progress in Polymer Science, vol. 37, no.7, pp.907-974, 2012.

[3] C. Gao, S. Zhang, L. Gao and M. Ding, "Microwave-assisted synthesis of highmolecular-weight poly(ether imide)s by phase-transfer catalysis", Journal of Applied Polymer Science, vol. 92, pp. 2415- 2419, 2004.

[4] L. Yi, C. Li, W. Huang, and D. Yan, "Soluble and transparent polyimides with high $\mathrm{Tg}$ from a new diamine containing tert-butyl and fluorene units", Journal of Polymer Science Part A: Polymer Chemistry, vol. 54, pp. 976-984, 2016.

[5] A. Kausar, S. Zulfiqar, Z. Ahmad, and M. I. Sarwar, "Novel processable and heat resistant poly(phenylthiourea azomethine imide)s: Synthesis and characterization", Polymer Degradation and Stability, vol.95, pp. 1826-1833, 2010.

[6] S.-H. Hsiao, C.-P. Yang, Y.-C. Chen, H.-M. Wang, and W. Guo, "Synthesis and properties of poly(ether imide)s derived from 2,5bis(3,4-dicarboxyphenoxy)biphenyl dianhydride and aromatic ether?diamines", Journal of Applied Polymer Science, vol. 113, pp. 39934002, 2009.

[7] J. P. Critchley, P. A. Grattan, M. A. White, and J. S. Pippett, "Perfluoroalkylene-linked aromatic polyimides. I. Synthesis, structure, and some general physical characteristics", Journal of Polymer Science Part A-1: Polymer Chemistry, vol. 10, pp. 1789-1807, 1972.

[8] T. P. Bender and Z. Y. Wang, "Synthesis of polyimides and segmented block copolyimides by transimidization", Journal of Polymer Science Part A: Polymer Chemistry, vol. 38, pp. 39913996, 2000.

[9] Y. Watanabe, Y. Sakai, Y. Shibasaki, S. Ando, M. Ueda, Y. Oishi, et al., "Synthesis of Wholly Alicyclic Polyimides from N-Silylated Alicyclic Diamines and Alicyclic Dianhydrides", Macromolecules, vol. 35, pp. 2277-2281, 2002.

[10] J X.-Q. Liu, M. Jikei, and M.-a. Kakimoto, "Synthesis and Properties of AB-
Type Semicrystalline Polyimides Prepared from Polyamic Acid Ethyl Ester Precursors,"Macromolecules, vol. 34, pp. 3146-3154, 2001.

[11] M. E. Pérez, D. M. Ruiz, M. Schneider, J. C. Autino, G. Romanelli, "La química verde como fuente de nuevos compuestos para el control de plagas agrícolas", Ciencia en Desarrollo, vol. 4, no.2, pp. 83-91, 2013.

[12] H. R. Kricheldorf, G. Schwarz, and S.-C. Fan, Çyclic Polyimides - A Comparison of Synthetic Methods", High Performance Polymers, vol. 16, pp. 543-555, 2004.

[13] X. L. Meng, L. Jiang, Y. D. Huang, Çharacterization and Thermal Degradation of Polyimide Derived from 5-amino-2(p-aminophenyl) Benzoxazole Monomer with Pyromellitic Dianhydride", Advanced Materials Research, vol. 455456, pp. 806-814, 2012.

[14] L. B. Nohara, A. M. Kawamoto, M. F. K. Takahashi, M. Wills, E. L. Nohara, and M. C. Rezende, "Síntese de um poli (ácido âmico) para aplicação como interfase em compósitos termoplásticos de alto desempenho", Polímeros, vol. 14, pp. 122-128, 2004.

[15] J. O. Iroh and W. Yuan, "Surface properties of carbon fibres modified by electrodeposition of polyamic acid", Polymer, vol. 37, pp. 41974203, 1996.

[16] Y. Pae, "Preparation and characterization of polyimide-g-nylon 6 copolymers from nonfunctionalized polyimides", Journal of Applied Polymer Science, vol. 99, pp. 292-299, 2006.

[17] S. Niyogi, S. Maiti, and B. Adhikari, "Modification of a polyimide film by blending with nylon 6. Its characterization and thermal property evaluation", European Polymer Journal, vol. 37, pp. 2079-2089, 2001.

[18] L. M. Moreno, E. Muñoz Prieto, H. Casanova, "Flocculation with Chitosan of Microalgae Native of the Colombian Plateau", Ciencia en Desarrollo, vol. 6, no.1, pp. 25-32, 2015.

[19] E. Muñoz Prieto, Bernabé Rivas, Julio Sánchez, "Natural Polymer Grafted with Synthetic Monomer by Microwave for Water Treatment - A Review", Ciencia en Desarrollo, vol. 4, no.1, pp. 219-240, 2012 